\title{
JOINT APPROXIMATION BY DIRICHLET L-FUNCTIONS
}

\author{
Antanas LaurinČIKas* — Darius ŠIAUČİUNAS**,c \\ (Communicated by István Gaál)
}

\begin{abstract}
In the paper, collections of analytic functions are simultaneously approximated by collections of shifts of Dirichlet $L$-functions $\left(L\left(s+\mathrm{i} \gamma_{1}(\tau), \chi_{1}\right), \ldots, L\left(s+\mathrm{i} \gamma_{r}(\tau), \chi_{r}\right)\right)$, with arbitrary Dirichlet characters $\chi_{1}, \ldots, \chi_{r}$. The differentiable functions $\gamma_{1}(\tau), \ldots, \gamma_{r}(\tau)$ and their derivatives satisfy certain growth conditions. The obtained results extend those of [PAŃKOWSKI, Ł.: Joint universality for dependent L-functions, Ramanujan J. 45 (2018), 181-195].
\end{abstract}

\section{Introduction}

Let $\chi$ be a Dirichlet character modulo $q \in \mathbb{N}$, i.e., $\chi$ is a function on $\mathbb{Z}, \chi(m) \not \equiv 0, \chi(m)=0$ for $(m, q)>1, \chi\left(m_{1} m_{2}\right)=\chi\left(m_{1}\right) \chi\left(m_{2}\right)$ for $m_{1}, m_{2} \in \mathbb{Z}$ and $\chi(m+q)=\chi(m)$ for all $m \in \mathbb{Z}$. The properties of Dirichlet characters can be found, for example, in 9 . The Dirichlet $L$-function $L(s, \chi), s=\sigma+\mathrm{i}$, is defined, for $\sigma>1$, by

$$
L(s, \chi)=\sum_{m=1}^{\infty} \frac{\chi(m)}{m^{s}}=\prod_{p}\left(1-\frac{\chi(p)}{p^{-s}}\right)^{-1}
$$

where the product is taken over all prime numbers. If $\chi(m)$ is the principal character $(\chi(m)=1$ if $(m, q)=1)$, then

$$
L(s, \chi)=\zeta(s) \prod_{p \mid q}\left(1-\frac{1}{p^{s}}\right),
$$

where $\zeta(s)$ is the Riemann zeta-function,

$$
\zeta(s)=\sum_{m=1}^{\infty} \frac{1}{m^{s}}=\prod_{p}\left(1-\frac{1}{p^{s}}\right)^{-1}, \quad \sigma>1 .
$$

Therefore, in this case, the function $L(s, \chi)$ has analytic continuation to the whole complex plane, except for the point $s=1$ which is a simple pole with residue $\prod_{p \mid q}(1-1 / p)$. If $\chi(m)$ is a non-principal character, then $L(s, \chi)$ has analytic continuation to an entire function.

Voronin discovered the universality property of the functions $L(s, \chi)$ concerning the approximation of analytic functions defined in the strip $D=\{s \in \mathbb{C}: 1 / 2<\sigma<1\}$. He proved [21] that if

2020 Mathematics Subject Classification: $11 \mathrm{M} 41$.

Keywords: Dirichlet $L$-functions, joint universality, functional independence, weak convergence.

The research of the first author is funded by the European Social Fund (project No. 09.3.3-LMT-K-712-01-0037) under grant agreement with the Research Council of Lithuania (LMT LT).

${ }^{\mathbf{c}}$ Corresponding author. 
$f(s)$ is a continuous non-vanishing function on the disc $|s| \leq r, 0<r<1 / 4$, and analytic in the interior of this disc, then, for every $\varepsilon>0$, there exists a real number $\tau=\tau(\varepsilon)$ such that

$$
\max _{|s| \leq r}|L(s+3 / 4+\mathrm{i} \tau, \chi)-f(s)|<\varepsilon .
$$

Moreover, Voronin in 22 considered the functional independence of Dirichlet $L$-functions with pairwise non-equivalent Dirichlet characters $\chi_{1}, \ldots, \chi_{r}$, and, for this, he in fact obtained in a nonexplicit form a joint universality theorem for $L\left(s, \chi_{1}\right), \ldots, L\left(s, \chi_{r}\right)$. Voronin's investigations were continued by Gonek [7, Bagchi [1,2] and the first author [11]. For the modern version of a joint universality theorem, we need some notation. Denote by $\mathcal{K}$ the class of compact subsets of the strip $D$ with connected complements, and by $H_{0}(K)$ with $K \in \mathcal{K}$ the class of continuous non-vanishing functions on $K$ that are analytic in the interior of $K$. Let meas $A$ be the Lebesgue measure of a measurable set $A \subset \mathbb{R}$. Then the following statement is true, see, for example, 19].

TheOREM 1.1. Let $\chi_{1}, \ldots, \chi_{r}$ be pairwise non-equivalent Dirichlet characters. For $j=1, \ldots, r$, let $K_{j} \in \mathcal{K}$, and let $f_{j}(s) \in H_{0}\left(K_{j}\right)$. Then for every $\varepsilon>0$,

$$
\liminf _{T \rightarrow \infty} \frac{1}{T} \operatorname{meas}\left\{\tau \in[0, T]: \sup _{1 \leq j \leq r} \sup _{s \in K_{j}}\left|L\left(s+\mathrm{i} \tau, \chi_{j}\right)-f_{j}(s)\right|<\varepsilon\right\}>0 .
$$

We recall that if $\chi(m)$ for $(m, q)=1$ has a period less than $q$, then the character $\chi$ is nonprimitive. In opposite case, $\chi$ is primitive. Every non-primitive character $\chi$ is induced by a primitive character, i.e., there exists a primitive character $\chi_{1}$ modulo $q_{1}, q_{1} \mid q$, such that

$$
\chi(m)= \begin{cases}\chi_{1}(m) & \text { if }\left(m, q_{1}\right)=1, \\ 0 & \text { otherwise. }\end{cases}
$$

Two Dirichlet characters are called non-equivalent if they are not induced by the same primitive character.

It is possible to consider the approximation of the collection $\left(f_{1}(s), \ldots, f_{r}(s)\right)$ by more general shifts $\left(L\left(s+\mathrm{i} \gamma_{1}(\tau), \chi_{1}\right), \ldots, L\left(s+\mathrm{i} \gamma_{r}(\tau), \chi_{r}\right)\right)$. Let $K_{1}=\cdots=K_{r}=K$. Then it follows from 8] that, under hypotheses of Theorem 1.1 , for every $\varepsilon>0$,

$$
\liminf _{T \rightarrow \infty} \frac{1}{T} \operatorname{meas}\left\{\tau \in[0, T]: \sup _{1 \leq j \leq r} \sup _{s \in K}\left|L\left(s+\mathrm{i} \gamma_{j}(\tau), \chi_{j}\right)-f_{j}(s)\right|<\varepsilon\right\}>0,
$$

where $\gamma_{j}(\tau)=\tau+\lambda_{j}$, with $K$ satisfying $\hat{K}_{k} \cap \hat{K}_{l}=\emptyset, k \neq l$, where $\hat{K}_{j}=\left\{s+\mathrm{i} \lambda_{j}: s \in K\right\}$, $j=1, \ldots, r$. Nakamura [14] obtained the inequality

$$
\liminf _{T \rightarrow \infty} \frac{1}{T} \operatorname{meas}\left\{\tau \in[0, T]: \sup _{1 \leq j \leq r} \sup _{s \in K}\left|L\left(s+\mathrm{i} \gamma_{j}(\tau), \chi\right)-f_{j}(s)\right|<\varepsilon\right\}>0,
$$

where $\gamma_{j}(\tau)=a_{j} \tau$ with algebraic numbers $a_{1}, \ldots, a_{r} \in \mathbb{R}$ linearly independent over the field of rational numbers $\mathbb{Q}$. The case $r=2$ was studied in [15] 17 with $a_{1}, a_{2} \in \mathbb{R} \backslash\{0\}, a_{1} \neq \pm a_{2}$.

The most general result belongs to Pańkowski 18. He proved the following theorem.

THEOREM 1.2. Suppose that $\chi_{1}, \ldots, \chi_{r}$ are Dirichlet characters, $\alpha_{1}, \ldots, \alpha_{r} \in \mathbb{R}, a_{1}, \ldots, a_{r} \in \mathbb{R}^{+}$, and $b_{1}, \ldots, b_{r}$ are such that

$$
b_{j} \in \begin{cases}\mathbb{R} & \text { if } \quad a_{j} \notin \mathbb{N}, \\ (-\infty, 0] \cup(1+\infty) & \text { if } \quad a_{j} \in \mathbb{N},\end{cases}
$$

and $a_{j} \neq a_{k}$ or $b_{j} \neq b_{k}$ if $k \neq j$. Moreover, let $K \in \mathcal{K}, f_{1}, \ldots, f_{r} \in H_{0}(K)$. Then, for every $\varepsilon>0$,

$$
\liminf _{T \rightarrow \infty} \frac{1}{T} \text { meas }\left\{\tau \in[2, T]: \sup _{1 \leq j \leq r} \sup _{s \in K}\left|L\left(s+\mathrm{i} \alpha_{j} \tau^{a_{j}} \log ^{b_{j}} \tau, \chi_{j}\right)-f_{j}(s)\right|<\varepsilon\right\}>0 .
$$


It is very important to stress that, in Theorem $1.2, \chi_{1}, \ldots, \chi_{r}$ are arbitrary, not necessarily pairwise non-equivalent, Dirichlet characters. The proof is based on the uniform distribution modulo 1.

Our aim is to obtain the joint universality for Dirichlet $L$-functions with other functions $\gamma_{j}(\tau)$ without using the uniform distribution theory. Moreover, we approximate in different sets $K_{1}, \ldots, K_{r} \in \mathcal{K}$.

Suppose that, for $j=1, \ldots, r, \gamma_{j}(\tau)$ is an increasing to infinity real continuously differentiable functions on $\left[T_{0}, \infty\right), T_{0}>0$, with derivative

(i)

where $\hat{\gamma}_{j}(\tau)$ is monotonic such that

$$
\gamma_{j}^{\prime}(\tau)=\hat{\gamma}_{j}(\tau)(1+o(1))
$$

and

$$
\hat{\gamma}_{1}(\tau)=o\left(\hat{\gamma}_{2}(\tau)\right), \ldots, \hat{\gamma}_{r-1}(\tau)=o\left(\hat{\gamma}_{r}(\tau)\right)
$$

(iii)

as $\tau \rightarrow \infty$.

$$
\gamma_{j}(2 \tau) \max _{\tau \leq u \leq 2 \tau} \frac{1}{\gamma_{j}^{\prime}(u)} \ll \tau
$$

Denote the class of $r$-tuples $\left(\gamma_{1}, \ldots, \gamma_{r}\right)$ satisfying the above hypotheses by $U_{r}$. Then the following joint universality theorem for Dirichlet $L$-functions is valid.

TheOREM 1.3. Suppose that $\chi_{1}, \ldots, \chi_{r}$ are arbitrary Dirichlet characters, and $\left(\gamma_{1}, \ldots, \gamma_{r}\right) \in U_{r}$. Let, for $j=1, \ldots, r, K_{j} \in \mathcal{K}$ and $f_{j} \in H_{0}\left(K_{j}\right)$. Then, for every $\varepsilon>0$,

$$
\liminf _{T \rightarrow \infty} \frac{1}{T-T_{0}} \operatorname{meas}\left\{\tau \in\left[T_{0}, T\right]: \sup _{1 \leq j \leq r} \sup _{s \in K_{j}}\left|L\left(s+\mathrm{i} \gamma_{j}(\tau), \chi_{j}\right)-f_{j}(s)\right|<\varepsilon\right\}>0 .
$$

Moreover, the limit

$$
\lim _{T \rightarrow \infty} \frac{1}{T-T_{0}} \text { meas }\left\{\tau \in\left[T_{0}, T\right]: \sup _{1 \leq j \leq r} \sup _{s \in K_{j}}\left|L\left(s+\mathrm{i} \gamma_{j}(\tau), \chi_{j}\right)-f_{j}(s)\right|<\varepsilon\right\}>0
$$

exists for all but at most countably many $\varepsilon>0$.

For example, the system of polynomials $\gamma_{1}(\tau)=\tau+1, \gamma_{2}(\tau)=\tau^{2}+\tau+1, \ldots, \gamma_{r}(\tau)=\tau^{r}+$ $\tau^{r-1}+\cdots+1$ is a member of the class $U_{r}$. Also $\left(\tau \log \tau, \ldots, \tau^{r} \log \tau\right) \in U_{r}$ and $\left(\tau\left(\Gamma^{\prime}(\tau) / \Gamma(\tau)\right), \ldots\right.$, $\left.\tau^{r}\left(\Gamma^{\prime}(\tau) / \Gamma(\tau)\right)\right) \in U_{r}$, where $\Gamma(\cdot)$ is the Euler gamma-function. We note that $\left(\tau \log \tau, \ldots, \tau^{r} \log \tau\right)$ does not satisfy hypotheses of Theorem 1.2 .

Denote by $H(D)$ the space of analytic on $D$ functions endowed with the topology of uniform convergence on compacta. Theorem 1.3 can be generalized for some compositions. We will give only one theoretical example. Denote by $H(K)$ with $K \in \mathcal{K}$ the class of continuous functions on $K$ that are analytic in the interior of $K$. Thus, $H_{0}(K) \subset H(K)$. Let $S=\{g \in H(D): g(s) \neq$ 0 or $g(s) \equiv 0\}$.

THEOREM 1.4. Suppose that $\left(\gamma_{1}, \ldots, \gamma_{r}\right) \in U_{r}$ and $F: H^{r}(D) \rightarrow H(D)$ is a continuous operator such that, for every open set $G \subset H(D)$, the set $\left(F^{-1} G\right) \cap S^{r}$ is non-empty. Let $K \in \mathcal{K}$ and $f \in H(K)$. Then, for every $\varepsilon>0$,

$$
\begin{aligned}
\liminf _{T \rightarrow \infty} \frac{1}{T-T_{0}} \operatorname{meas}\left\{\tau \in\left[T_{0}, T\right]:\right. & \\
& \left.\sup _{s \in K}\left|F\left(L\left(s+\mathrm{i} \gamma_{1}(\tau), \chi_{1}\right), \ldots, L\left(s+\mathrm{i} \gamma_{r}(\tau), \chi_{r}\right)\right)-f(s)\right|<\varepsilon\right\}>0 .
\end{aligned}
$$


Moreover, the limit

$$
\begin{aligned}
\lim _{T \rightarrow \infty} \frac{1}{T-T_{0}} \operatorname{meas}\left\{\tau \in\left[T_{0}, T\right]:\right. \\
\left.\qquad \sup _{s \in K}\left|F\left(L\left(s+\mathrm{i} \gamma_{1}(\tau), \chi_{1}\right), \ldots, L\left(s+\mathrm{i} \gamma_{r}(\tau), \chi_{r}\right)\right)-f(s)\right|<\varepsilon\right\}>0
\end{aligned}
$$

exists for all but at most countably many $\varepsilon>0$.

It is known that the sets of values taking by zeta or $L$-functions are in some sense dense. First, Bohr [4] obtained that the function $\zeta(s)$ takes every non-zero value infinitely many times in the strip $\{s \in \mathbb{C}: 1<\sigma<1+\delta\}$ for any $\delta>0$. Bohr and Courant [5] obtained that, for any fixed $\sigma$, $1 / 2<\sigma<1$, the set

$$
\{\zeta(\sigma+\mathrm{i} t): t \in \mathbb{R}\}
$$

is dense in $\mathbb{C}$. Voronin extended and generalized the above results. He proved [20] that the set

$$
\left\{\left(\zeta\left(s_{1}+\mathrm{i} \tau\right), \ldots, \zeta\left(s_{n}+\mathrm{i} \tau\right)\right): \tau \in \mathbb{R}\right\}
$$

with any fixed different $s_{1}, \ldots, s_{n}, 1 / 2<\operatorname{Re} s_{k}<1,1 \leq k \leq n$, and the set

$$
\left\{\left(\zeta(\sigma+\mathrm{i} t), \zeta^{\prime}(\sigma+\mathrm{i} t), \ldots, \zeta^{(n-1)}(\sigma+\mathrm{i} t)\right): t \in \mathbb{R}\right\}
$$

with every fixed $\sigma, 1 / 2<\sigma<1$, are dense in $\mathbb{C}^{n}$. Moreover, Voronin obtained a joint generalization of the later result for Dirichlet $L$-functions. Namely, he proved 22 that if $\chi_{1}, \ldots, \chi_{r}$ are pairwise non-equivalent Dirichlet characters, then the set

$$
\begin{gathered}
\left\{\left(L\left(\sigma+\mathrm{i} t, \chi_{1}\right), L^{\prime}\left(\sigma+\mathrm{i} t, \chi_{1}\right), \ldots, L^{(n-1)}\left(\sigma+\mathrm{i} t, \chi_{1}\right), \ldots,\right.\right. \\
\left.\left.L\left(\sigma+\mathrm{i} t, \chi_{r}\right), L^{\prime}\left(\sigma+\mathrm{i} t, \chi_{r}\right), \ldots, L^{(n-1)}\left(\sigma+\mathrm{i} t, \chi_{r}\right)\right) t \in \mathbb{R}\right\}
\end{gathered}
$$

is everywhere dense in $\mathbb{C}^{r \times n}$ for every fixed $\sigma, 1 / 2<\sigma<1$.

Theorem 1.3 has the following corollary.

Corollary 1.4.1. Suppose that $\chi_{1}, \ldots, \chi_{r}$ are arbitrary Dirichlet characters, and $\left(\gamma_{1}, \ldots, \gamma_{r}\right) \in U_{r}$. Then, for every fixed $\sigma, 1 / 2<\sigma<1$, the set

$$
\begin{aligned}
& \left\{\left(L\left(\sigma+\mathrm{i} \gamma_{1}(t), \chi_{1}\right), L^{\prime}\left(\sigma+\mathrm{i} \gamma_{1}(t), \chi_{1}\right), \ldots, L^{(n-1)}\left(\sigma+\mathrm{i} \gamma_{1}(t), \chi_{1}\right), \ldots,\right.\right. \\
& \left.\left.\quad L\left(\sigma+\mathrm{i} \gamma_{r}(t), \chi_{r}\right), L^{\prime}\left(\sigma+\mathrm{i} \gamma_{r}(t), \chi_{r}\right), \ldots, L^{(n-1)}\left(\sigma+\mathrm{i} \gamma_{r}(t), \chi_{r}\right)\right): t \geq T_{0}\right\}
\end{aligned}
$$

is everywhere dense in $\mathbb{C}^{r \times n}$.

The proof of the corollary uses Theorem 1.3 and repeats Voronin's arguments.

Corollary 1.4.1 implies the following functional independence property of Dirichlet $L$-functions.

Corollary 1.4.2. Suppose that $\chi_{1}, \ldots, \chi_{r}$ are arbitrary Dirichlet characters, $\Phi: \mathbb{C}^{r \times n} \rightarrow \mathbb{C}$ is a continuous function, and

$$
\Phi\left(L\left(s, \chi_{1}\right), L^{\prime}\left(s, \chi_{1}\right), \ldots, L^{(n-1)}\left(s, \chi_{1}\right), \ldots, L\left(s, \chi_{r}\right), L^{\prime}\left(s, \chi_{r}\right), \ldots, L^{(n-1)}\left(s, \chi_{r}\right)\right)=0
$$

identically for $s$. Then $\Phi \equiv 0$.

For the proof of universality theorems, we apply a method different from that of [18]. This method is probabilistic, and is based on weak convergence of probability measures in the space of analytic functions, see $1,10,12$ and 19. 


\section{Lemmas}

Denote by $\mathcal{B}(\mathbb{X})$ the Borel $\sigma$-field of the space $\mathbb{X}$, and by $\gamma$ the unit circle on the complex plane. Define the set

$$
\Omega=\prod_{p \in \mathbb{P}} \gamma_{p}
$$

where $\mathbb{P}$ is the set of all prime numbers, and $\gamma_{p}=\gamma$ for all $p \in \mathbb{P}$. With product topology and pointwise multiplication, the infinite-dimensional torus $\Omega$ is a compact topological Abelian group. Let

$$
\Omega^{r}=\Omega_{1} \times \cdots \times \Omega_{r},
$$

where $\Omega_{j}=\Omega$ for $j=1, \ldots, r$. Then, again, $\Omega^{r}$ is a compact topological Abelian group. Therefore, on $\left(\Omega^{r}, \mathcal{B}\left(\Omega^{r}\right)\right)$, the probability Haar measure $m_{H}^{r}$ exists. This gives the probability space $\left(\Omega^{r}, \mathcal{B}\left(\Omega^{r}\right), m_{H}^{r}\right)$. For $j=1, \ldots, r$, denote by $\omega_{j}(p)$ the $p$ th component of an element $\omega_{j} \in \Omega_{j}$, $p \in \mathbb{P}$. Let $\omega=\left(\omega_{1}, \ldots, \omega_{r}\right), \omega_{j} \in \Omega_{j}$, be the elements of $\Omega^{r}$.

For $A \in \mathcal{B}\left(\Omega^{r}\right)$, define

$$
Q_{T}^{r}(A)=\frac{1}{T-T_{0}} \text { meas }\left\{\tau \in\left[T_{0}, T\right]:\left(\left(p^{-\mathrm{i} \gamma_{1}(\tau)}: p \in \mathbb{P}\right), \ldots,\left(p^{-\mathrm{i} \gamma_{r}(\tau)}: p \in \mathbb{P}\right)\right) \in A\right\} .
$$

Lemma 2.1 (Main lemma). Suppose that $\left(\gamma_{1}, \ldots, \gamma_{r}\right) \in U_{r}$. Then $Q_{T}^{r}$ converges weakly to the Haar measure $m_{H}^{r}$ as $T \rightarrow \infty$.

Proof. Let $g_{Q_{T}^{r}}\left(\underline{k}_{1}, \ldots, \underline{k}_{r}\right), \underline{k}_{j}=\left(k_{j p}: k_{j p} \in \mathbb{Z}, p \in \mathbb{P}\right), j=1, \ldots, r$, be the Fourier transform of $Q_{T}^{r}$, i.e.,

$$
g_{Q_{T}^{r}}\left(\underline{k}_{1}, \ldots, \underline{k}_{r}\right)=\int_{\Omega^{r}}\left(\prod_{j=1}^{r} \prod_{p \in \mathbb{P}}^{\prime} \omega^{k_{j p}}(p)\right) \mathrm{d} Q_{T}^{r},
$$

where the sign "' " means that only a finite number of integers $k_{j p}, j=1, \ldots, r$, are distinct from zero. Thus, by the definition of $Q_{T}^{r}$,

$$
g_{Q_{T}^{r}}\left(\underline{k}_{1}, \ldots, \underline{k}_{r}\right)=\frac{1}{T-T_{0}} \int_{T_{0}}^{T} \exp \left\{-\mathrm{i} \sum_{j=1}^{r} \gamma_{j}(\tau) \sum_{p \in \mathbb{P}}^{\prime} k_{j p} \log p\right\} \mathrm{d} \tau .
$$

Let, for brevity,

$$
a_{j}=\sum_{p \in \mathbb{P}}^{\prime} k_{j p} \log p, \quad j=1, \ldots, r .
$$

Since the set $\{\log p: p \in \mathbb{P}\}$ is linearly independent over the field of rational numbers $\mathbb{Q}, a_{j}=0$ if and only if $\underline{k}_{j}=\underline{0}, j=1, \ldots, r$. Clearly, in view of 2.1 ,

$$
g_{Q_{T}^{r}}(\underline{0}, \ldots, \underline{0})=1 .
$$

Now, suppose that $\left(\underline{k}_{1}, \ldots, \underline{k}_{r}\right) \neq(\underline{0}, \ldots, \underline{0})$. Since $\left(\gamma_{1}, \ldots, \gamma_{r}\right) \in U_{r}$, we have

$$
\left(\sum_{j=1}^{r} a_{j} \gamma_{j}(\tau)\right)^{\prime}=\sum_{j=1}^{r} a_{j} \gamma_{j}^{\prime}(\tau)=\sum_{j=1}^{r} a_{j} \hat{\gamma}_{j}(\tau)(1+o(1))=a_{j_{0}} \hat{\gamma}_{j_{0}}(\tau)(1+o(1))
$$

as $\tau \rightarrow \infty$, where $j_{0}=\max \left(j: a_{j} \neq 0\right)$. Hence,

$$
\left(\sum_{j=1}^{r} a_{j} \gamma_{j}^{\prime}(\tau)\right)^{-1}=\frac{1}{a_{j_{0}} \hat{\gamma}_{j_{0}}(\tau)(1+o(1))}=\frac{1}{a_{j_{0}} \hat{\gamma}_{j_{0}}(\tau)}(1+o(1))
$$


as $\tau \rightarrow \infty$. Moreover, since $\gamma_{j}(\tau) \rightarrow \infty$ as $\tau \rightarrow \infty$, we have, in view of (iii) of the class $U_{r}$, that

$$
\frac{1}{\hat{\gamma}_{j}(\tau)}=o(\tau)
$$

as $\tau \rightarrow \infty, j=1, \ldots, r$. Let $A(\tau)=\sum_{j=1}^{r} a_{j} \gamma_{j}(\tau)$. Then 2.3), 2.4 , the monotonicity of $\hat{\gamma}_{j}(\tau)$, and the second mean value theorem show that

$$
\begin{aligned}
\int_{T_{0}}^{T} \cos A(\tau) \mathrm{d} \tau & =\int_{\log T}^{T} \cos A(\tau) \mathrm{d} \tau+O(\log T)=\int_{\log T}^{T} \frac{1}{A^{\prime}(\tau)} \cos A(\tau) \mathrm{d} A(\tau)+O(\log T) \\
& =\int_{\log T}^{T} \frac{1}{a_{j_{0}} \hat{\gamma}_{j_{0}}(\tau)} \cos A(\tau) \mathrm{d} A(\tau)+\int_{\log T}^{T} \frac{o(1)}{a_{j_{0}} \hat{\gamma}_{j_{0}}(\tau)} \cos A(\tau) \mathrm{d} A(\tau)+O(\log T) \\
& =\int_{\log T}^{T} \frac{1}{a_{j_{0}} \hat{\gamma}_{j_{0}}(\tau)} \mathrm{d}(\sin A(\tau))+\int_{\log T}^{T} \frac{o(1)(1+o(1))}{A^{\prime}(\tau)} \cos A(\tau) \mathrm{d} A(\tau)+O(\log T) \\
& =o(T)+\int_{\log T}^{T} o(1) \cos A(\tau) \mathrm{d} \tau+O(\log T)=o(T)
\end{aligned}
$$

as $T \rightarrow \infty$. Similarly, we find that

$$
\int_{T_{0}}^{T} \sin A(\tau) \mathrm{d} \tau=o(T)
$$

as $T \rightarrow \infty$. This, 2.5 and 2.1) show that, in the case $\left(\underline{k}_{1}, \ldots, \underline{k}_{r}\right) \neq(\underline{0}, \ldots, \underline{0})$,

$$
g_{Q_{T}^{r}}\left(\underline{k}_{1}, \ldots, \underline{k}_{r}\right)=o(1), \quad T \rightarrow \infty .
$$

Thus, in view of 2.2,

$$
\lim _{T \rightarrow \infty} g_{Q_{T}^{r}}\left(\underline{k}_{1}, \ldots, \underline{k}_{r}\right)= \begin{cases}1 & \text { if }\left(\underline{k}_{1}, \ldots, \underline{k}_{r}\right)=(\underline{0}, \ldots, \underline{0}), \\ 0 & \text { if }\left(\underline{k}_{1}, \ldots, \underline{k}_{r}\right) \neq(\underline{0}, \ldots, \underline{0}) .\end{cases}
$$

Since the right-hand side of the latter equality is the Fourier transform of the Haar measure $m_{H}^{r}$, the lemma follows by a continuity theorem for probability measures on compact groups.

Lemma 2.1. by a standard way, implies a joint limit theorem in the space $H^{r}(D)$ for absolutely convergent Dirichlet series. Let $\sigma_{0}>1 / 2$ be a fixed number, $\underline{\chi}=\left(\chi_{1}, \ldots, \chi_{r}\right)$, for $m, n \in \mathbb{N}$,

$$
v_{n}(m)=\exp \left\{-\left(\frac{m}{n}\right)^{\sigma_{0}}\right\}
$$

and

$$
\underline{L}_{n}(s, \underline{\chi})=\left(L_{n}\left(s, \chi_{1}\right), \ldots, L_{n}\left(s, \chi_{r}\right)\right),
$$

where

$$
L_{n}\left(s, \chi_{j}\right)=\sum_{m=1}^{\infty} \frac{\chi_{j}(m) v_{n}(m)}{m^{s}}, \quad j=1, \ldots, r
$$




\section{JOINT APPROXIMATION BY DIRICHLET L-FUNCTIONS}

and

$$
\underline{L}_{n}(s, \omega, \underline{\chi})=\left(L_{n}\left(s, \omega_{1}, \chi_{1}\right), \ldots, L_{n}\left(s, \omega_{r}, \chi_{r}\right)\right)
$$

where

$$
L_{n}\left(s, \omega_{j}, \chi_{j}\right)=\sum_{m=1}^{\infty} \frac{\chi_{j}(m) \omega_{j}(m) v_{n}(m)}{m^{s}}, \quad j=1, \ldots, r
$$

and, for $m \in \mathbb{N}$,

$$
\omega_{j}(m)=\prod_{\substack{p^{l} \mid m \\ p^{l+1} \nmid m}} \omega_{j}^{l}(p), \quad j=1, \ldots, r .
$$

Then the series for $L_{n}\left(s, \chi_{j}\right)$ and $L\left(s, \omega_{j}, \chi_{j}\right)$ are absolutely convergent for $\sigma>1 / 2, j=1, \ldots, r$. Define the function $u_{n}: \Omega^{r} \rightarrow H^{r}(D)$ by the formula

$$
u_{n}(\omega)=\underline{L}_{n}(s, \omega, \underline{\chi}), \quad \omega \in \Omega^{r} .
$$

The absolute convergence of the series for $L_{n}\left(s, \omega_{j}, \chi_{j}\right), j=1, \ldots, r$, implies the continuity of the function $u_{n}$. Let $V_{n}=m_{H}^{r} u_{n}^{-1}$, where

$$
V_{n}(A)=m_{H}^{r} u_{n}^{-1}(A)=m_{H}^{r}\left(u_{n}^{-1} A\right), \quad A \in \mathcal{B}\left(H^{r}(D)\right) .
$$

For $A \in \mathcal{B}\left(H^{r}(D)\right)$, define

$$
P_{T, n}(A)=\frac{1}{T-T_{0}} \text { meas }\left\{\tau \in\left[T_{0}, T\right]: \underline{L}_{n}(s+\underline{\mathrm{i}} \underline{\gamma}(\tau), \underline{\chi}) \in A\right\},
$$

where $\underline{\gamma}(\tau)=\left(\gamma_{1}(\tau), \ldots, \gamma_{r}(\tau)\right)$ and

$$
\underline{L}_{n}(s+\underline{\mathrm{i}} \underline{(\tau)}, \underline{\chi})=\left(L_{n}\left(s+\mathrm{i} \gamma_{1}(\tau), \chi_{1}\right), \ldots, L_{n}\left(s+\mathrm{i} \gamma_{r}(\tau), \chi_{r}\right)\right) .
$$

Then Lemma 2.1, the continuity of $u_{n}$ and [3: Theorem 5.1] lead to the following statement.

Lemma 2.2. Suppose that $\left(\gamma_{1}, \ldots, \gamma_{r}\right) \in U_{r}$. Then $P_{T, n}$ converges weakly to the measure $V_{n}$ as $T \rightarrow \infty$.

The family of probability measures $\left\{V_{n}: n \in \mathbb{N}\right\}$ is very important for the investigation of the collection

$$
\underline{L}(s+\underline{\mathrm{i}} \underline{\gamma}(\tau), \underline{\chi})=\left(L\left(s+\mathrm{i} \gamma_{1}(\tau), \chi_{1}\right), \ldots, L\left(s+\mathrm{i} \gamma_{r}(\tau), \chi_{r}\right)\right) .
$$

We recall that the family of probability measures $\{P\}$ on $(\mathbb{X}, \mathcal{B}(\mathbb{X}))$ is tight if, for every $\varepsilon>0$, there exists a compact set $K=K(\varepsilon) \subset \mathbb{X}$ such that

$$
P(K)>1-\varepsilon
$$

for all $P \in\{P\}$.

Lемма 2.3. The family $\left\{V_{n}: n \in \mathbb{N}\right\}$ is tight.

Proof. For $j=1, \ldots, r$, let $m_{H, j}$ be the probability Haar measure on $\left(\Omega_{j}, \mathcal{B}\left(\Omega_{j}\right)\right)$, and $u_{n, j}$ : $\Omega_{j} \rightarrow H(D)$ be given by the formula

$$
u_{n, j}\left(\omega_{j}\right)=L_{n}\left(s, \omega_{j}, \chi_{j}\right) .
$$


Then $V_{n, j}=m_{H, j} u_{n, j}^{-1}, j=1, \ldots, r$, are the marginal measures of $V_{n}$. Actually, for $A \in \mathcal{B}(H(D))$,

$$
\begin{aligned}
V_{n} & (\underbrace{H(D) \times \cdots \times H(D)}_{j-1} \times A \times H(D) \times \cdots \times H(D)) \\
& =m_{H}^{r} u_{n}^{-1}(\underbrace{H(D) \times \cdots \times H(D)}_{j-1} \times A \times H(D) \times \cdots \times H(D)) \\
& =m_{H}^{r}\left(u_{n}^{-1}(\underbrace{H(D) \times \cdots \times H(D)}_{j-1} \times A \times H(D) \times \cdots \times H(D))\right) \\
& =m_{H}^{r}\left(u_{n, j}^{-1} A\right)=m_{H, j} u_{n, j}^{-1}(A) .
\end{aligned}
$$

It is easy to see using the absolute convergence of the series for $L_{n}\left(s, \chi_{j}\right)$, see, for example, the proof of Lemma 4.11 from [19] for more general functions from the Selberg class, that the families $\left\{V_{n, j}: n \in \mathbb{N}\right\}, j=1, \ldots, r$, are tight. Therefore, for every $\varepsilon>0$, there exists a compact set $K_{j}=K_{j}(\varepsilon) \subset H(D)$ such that

$$
V_{n, j}\left(K_{j}\right)>1-\frac{\varepsilon}{r}, \quad j=1, \ldots, r
$$

for all $n \in \mathbb{N}$. The set $K=K_{1} \times \cdots \times K_{r}$ is compact in the space $H^{r}(D)$, and, in view of 2.6),

$$
\begin{aligned}
V_{n}\left(H^{r}(D) \backslash K\right) & =V_{n}\left(\bigcup_{j=1}^{r}(\underbrace{H(D) \times \cdots \times H(D)}_{j-1} \times\left(H(D) \backslash K_{j}\right) \times H(D) \times \cdots \times H(D))\right) \\
& \leq \sum_{j=1}^{r} V_{n, j}\left(H(D) \backslash K_{j}\right) \leq \varepsilon
\end{aligned}
$$

for all $n \in \mathbb{N}$. The lemma is proved.

\section{Mean square estimates}

Mean square estimates play an important role in the universality theory of zeta- and $L$-functions. In this section, we present estimates for generalized mean squares of Dirichlet $L$-functions.

Lemma 3.1. Suppose that $\left(\gamma_{1}, \ldots, \gamma_{r}\right) \in U_{r}$. Then, for fixed $\sigma, 1 / 2<\sigma<1$, and $t \in \mathbb{R}$,

$$
\int_{T_{0}}^{T}\left|L\left(\sigma+\mathrm{i} t+\mathrm{i} \gamma_{j}(\tau), \chi_{j}\right)\right|^{2} \mathrm{~d} \tau \ll_{\sigma} T(1+|t|), \quad j=1, \ldots, r .
$$

Pr o of. It is well known that, for fixed $\sigma, 1 / 2<\sigma<1$,

$$
\int_{T_{0}}^{T}\left|L\left(\sigma+\mathrm{i} t, \chi_{j}\right)\right|^{2} \mathrm{~d} t \ll_{\sigma} T
$$

Therefore, for all $t \in \mathbb{R}$,

$$
\int_{0}^{|t|+\gamma_{j}(\tau)}\left|L\left(\sigma+\mathrm{i} u, \chi_{j}\right)\right|^{2} \mathrm{~d} u \ll_{\sigma}\left(|t|+\gamma_{j}(\tau)\right) .
$$


Thus, for $X \geq T_{0}$,

$$
\begin{aligned}
\int_{X}^{2 X}\left|L\left(\sigma+\mathrm{i} t+\mathrm{i} \gamma_{j}(\tau), \chi_{j}\right)\right|^{2} \mathrm{~d} \tau & =\int_{X}^{2 X} \frac{1}{\gamma_{j}^{\prime}(\tau)}\left|L\left(\sigma+\mathrm{i} t+\mathrm{i} \gamma_{j}(\tau), \chi_{j}\right)\right|^{2} \mathrm{~d} \gamma_{j}(\tau) \\
& \ll \max _{X \leq \tau \leq 2 X} \frac{1}{\gamma_{j}^{\prime}(\tau)} \int_{X}^{2 X} \mathrm{~d}\left(\int_{0}^{t+\gamma_{j}(\tau)}\left|L\left(\sigma+\mathrm{i} u, \chi_{j}\right)\right|^{2} \mathrm{~d} u\right) \\
& \ll_{\sigma}\left(|t|+\gamma_{j}(2 X)\right) \max _{X \leq \tau \leq 2 X} \frac{1}{\gamma_{j}^{\prime}(\tau)} \ll_{\sigma} X(1+|t|)
\end{aligned}
$$

in virtue of properties of $U_{r}$. Now, taking $X=T 2^{-k-1}$ and summing over $k=0,1, \ldots$, we get the estimate of the lemma.

Lemma 3.1 allows to obtain the approximation in the mean for $\underline{L}(s, \underline{\chi})$ by $\underline{L}_{n}(s, \underline{\chi})$. For $g_{1}, g_{2} \in$ $H(D)$, let

$$
\rho\left(g_{1}, g_{2}\right)=\sum_{l=1}^{\infty} 2^{-l} \frac{\sup _{s \in K_{l}}\left|g_{1}(s)-g_{2}(s)\right|}{1+\sup _{s \in K_{l}}\left|g_{1}(s)-g_{2}(s)\right|},
$$

where $\left\{K_{l}: l \in \mathbb{N}\right\} \subset D$ is a sequence of compact sets such that

$$
D=\bigcup_{l=1}^{\infty} K_{l}
$$

$K_{l} \subset K_{l+1}$ for all $l \in \mathbb{N}$, and if $K \subset D$ is a compact set, then $K \subset K_{l}$ for some $l$. Then $\rho$ is a metric in $H(D)$ inducing the topology of uniform convergence on compacta. For $\underline{g}_{1}=\left(g_{11}, \ldots, g_{1 r}\right)$, $\underline{g}_{2}=\left(g_{21}, \ldots, g_{2 r}\right) \in H^{r}(D)$, define

$$
\underline{\rho}\left(\underline{g}_{1}, \underline{g}_{2}\right)=\max _{1 \leq j \leq r} \rho\left(g_{1 j}, g_{2 j}\right) .
$$

Then $\underline{\rho}$ is a metric in $H^{r}(D)$ inducing the product topology.

Lемма 3.2. The equality

$$
\lim _{n \rightarrow \infty} \limsup _{T \rightarrow \infty} \frac{1}{T-T_{0}} \int_{T_{0}}^{T} \underline{\rho}\left(\underline{L}(s+\underline{\mathrm{i}} \underline{\gamma}(\tau), \underline{\chi}), \underline{L}_{n}(s+\underline{\mathrm{i}} \underline{\gamma}(\tau), \underline{\chi})\right) \mathrm{d} \tau=0
$$

holds.

Proof. From the definitions of the metrics $\rho$ and $\underline{\rho}$, it follows that it suffices to prove that, for every compact set $K \subset D$ and all $j=1, \ldots, r$,

$$
\lim _{n \rightarrow \infty} \limsup _{T \rightarrow \infty} \frac{1}{T-T_{0}} \int_{T_{0}}^{T} \sup _{s \in K}\left|L\left(s+\mathrm{i} \gamma_{j}(\tau), \chi_{j}\right)-L_{n}\left(s+\mathrm{i} \gamma_{j}(\tau), \chi_{j}\right)\right| \mathrm{d} \tau=0 .
$$

Let

$$
l_{n}(s)=\frac{s}{\sigma_{0}} \Gamma\left(\frac{s}{\sigma_{0}}\right) n^{-s}
$$


where the number $\sigma_{0}$ is from the definition of $v_{n}(m)$. Then an application of the Mellin formula leads to the representation

$$
L_{n}(s, \chi)=\frac{1}{2 \pi \mathrm{i}} \int_{\sigma_{0}-\mathrm{i} \infty}^{\sigma_{0}+\mathrm{i} \infty} L(s+z, \chi) l_{n}(z) \frac{\mathrm{d} z}{z},
$$

where $\chi$ is an arbitrary Dirichlet character modulo $q$. Let $K$ be an arbitrary fixed compact set of the strip $D$. We fix $\varepsilon>0$ such that $1 / 2+2 \varepsilon \leq \sigma \leq 1-\varepsilon$ for points $s \in K$. The residue theorem for $\hat{\sigma}_{0}>0$ implies

$$
L_{n}(s, \chi)-L(s, \chi)=\frac{1}{2 \pi \mathrm{i}} \int_{-\hat{\sigma}_{0}-\mathrm{i} \infty}^{-\hat{\sigma}_{0}+\mathrm{i} \infty} L(s+z, \chi) l_{n}(z) \frac{\mathrm{d} z}{z}+R_{n}(s),
$$

where

$$
R_{n}(s)= \begin{cases}0 & \text { if } \chi \text { is a non-principal character } \\ \prod_{p \mid q}\left(1-\frac{1}{p}\right) \frac{l_{n}(1-s)}{1-s} & \text { otherwise. }\end{cases}
$$

Denote by $s=\sigma+\mathrm{i} v$ the points of $K$, and take

$$
\hat{\sigma}_{0}=\sigma-\varepsilon-\frac{1}{2}, \quad \sigma_{0}=\frac{1}{2}+\varepsilon .
$$

Let $\gamma(\tau)$ be one of the functions $\gamma_{j}(\tau), j=1, \ldots, r$. Then, by (3.2),

$$
\begin{aligned}
& \left|L_{n}(s+\mathrm{i} \gamma(\tau), \chi)-L(s+\mathrm{i} \gamma(\tau), \chi)\right| \\
& \quad \leq \frac{1}{2 \pi} \int_{-\infty}^{\infty}\left|L\left(s+\mathrm{i} \gamma(\tau)-\hat{\sigma}_{0}+\mathrm{i} t, \chi\right)\right| \frac{\left|l_{n}\left(-\hat{\sigma}_{0}+\mathrm{i} t\right)\right|}{\left|-\hat{\sigma}_{0}+\mathrm{i} t\right|} \mathrm{d} t+\mid R_{n}(s+\mathrm{i} \gamma(\tau) \mid \\
& \quad=\frac{1}{2 \pi} \int_{-\infty}^{\infty}|L(1 / 2+\varepsilon+\mathrm{i}(t+\gamma(\tau)), \chi)| \frac{\left|l_{n}(1 / 2+\varepsilon-s+\mathrm{i} t)\right|}{|1 / 2+\varepsilon-s+\mathrm{i} t|} \mathrm{d} t+\mid R_{n}(s+\mathrm{i} \gamma(\tau) \mid
\end{aligned}
$$

after a shift $t+v \rightarrow t$. Thus,

$$
\begin{aligned}
& \frac{1}{T-T_{0}} \int_{T_{0}}^{T} \sup _{s \in K}\left|L(s+\mathrm{i} \gamma(\tau), \chi)-L_{n}(s+\mathrm{i} \gamma(\tau), \chi)\right| \mathrm{d} \tau \\
& \quad \leq \frac{1}{2 \pi} \int_{-\infty}^{\infty}\left(\frac{1}{T-T_{0}} \int_{T_{0}}^{T}|L(1 / 2+\varepsilon+\mathrm{i}(t+\gamma(\tau)), \chi)| \mathrm{d} \tau\right) \sup _{s \in K} \frac{\left|l_{n}(1 / 2+\varepsilon-s+\mathrm{i} t)\right|}{|1 / 2+\varepsilon-s+\mathrm{i} t|} \mathrm{d} t \\
& \quad+\frac{1}{T-T_{0}} \int_{T_{0}}^{T} \sup _{s \in K} \mid R_{n}(s+\mathrm{i} \gamma(\tau) \mid \mathrm{d} \tau \\
& \quad \stackrel{\text { def }}{=} I_{1}+I_{2} .
\end{aligned}
$$

For the function $\Gamma(s)$, the well-known estimate

$$
\Gamma(\sigma+\mathrm{i} t) \ll \exp \{-c|t|\}, \quad c>0,
$$

60 
uniform in $\sigma_{1} \leq \sigma \leq \sigma_{2}$ is valid. Therefore, the definition of $l_{n}(s)$ implies the bound

$$
\frac{l_{n}(1 / 2+\varepsilon-s+\mathrm{i} t)}{1 / 2+\varepsilon-s+\mathrm{i} t} \ll \frac{n^{1 / 2+\varepsilon-\sigma}}{\sigma_{0}} \exp \left\{-\frac{c}{\sigma_{0}}|t-v|\right\} \ll_{K} n^{-\varepsilon} \exp \{-c|t|\} .
$$

Thus, by Lemma 3.1 .

$$
I_{1} \ll_{K, \varepsilon} n^{-\varepsilon} \int_{-\infty}^{\infty}\left(1+|t|^{1 / 2}\right) \exp \{-c|t|\} \mathrm{d} t \ll_{K, \varepsilon} n^{-\varepsilon} .
$$

Similarly, we find that

$$
\begin{aligned}
I_{2} & \ll_{K, q} n^{1 / 2-2 \varepsilon} \frac{1}{T-T_{0}} \int_{T_{0}}^{T} \exp \{-c \gamma(\tau)\} \mathrm{d} \tau \ll_{K, q} n^{1 / 2-2 \varepsilon}\left(\frac{\log T}{T}+\frac{1}{T} \int_{\log T}^{T} \exp \{-c \gamma(\tau)\} \mathrm{d} \tau\right) \\
& \ll_{K, q} n^{1 / 2-2 \varepsilon}\left(\frac{\log T}{T}+\frac{1}{T} \exp \left\{-\frac{c}{2} \gamma(\log T)\right\} \int_{\log T}^{T} \exp \left\{-\frac{c}{2} \gamma(\tau)\right\} \mathrm{d} \tau\right)=o(T)
\end{aligned}
$$

as $T \rightarrow \infty$ because $\gamma(\tau) \rightarrow \infty$ as $\tau \rightarrow \infty$. This, 3.4 and 3.3 prove 3.1. The lemma is proved.

\section{Limit theorem}

In this section, we consider the weak convergence for

$$
P_{T}(A) \stackrel{\text { def }}{=} \frac{1}{T-T_{0}} \text { meas }\left\{\tau \in\left[T_{0}, T\right]: \underline{L}(s+\underline{\mathrm{i}} \underline{\gamma}(\tau), \underline{\chi}) \in A\right\}, \quad A \in \mathcal{B}\left(H^{r}(D)\right),
$$

as $T \rightarrow \infty$. For this, we recall the useful property of convergence in distribution $(\stackrel{\mathcal{D}}{\rightarrow})$.

Proposition 4.1. Suppose that the space $(\mathbb{X}, d)$ is separable, and $X_{k n}$ and $X_{n}, k \in \mathbb{N}, n \in \mathbb{N}$, are $\mathbb{X}$-valued random elements defined on the same probability space with measure $\nu$. If $X_{k n} \underset{n \rightarrow \infty}{\stackrel{\mathcal{D}}{\longrightarrow}}$ $Z_{k} \underset{k \rightarrow \infty}{\stackrel{\mathcal{D}}{\longrightarrow}} X$ and, for every $\varepsilon>0$,

$$
\lim _{k \rightarrow \infty} \limsup _{n \rightarrow \infty} \nu\left\{d\left(X_{k n}, X_{n}\right) \geq \varepsilon\right\}=0,
$$

then $X_{n} \underset{n \rightarrow \infty}{\stackrel{\mathcal{D}}{\longrightarrow}} X$.

The proof of the proposition is given in 3 .

Define

where

$$
\underline{L}(s, \omega, \underline{\chi})=\left(L\left(s, \omega_{1}, \chi_{1}\right), \ldots, L\left(s, \omega_{r}, \chi_{r}\right)\right),
$$

$$
L\left(s, \omega_{j}, \chi_{j}\right)=\sum_{m=1}^{\infty} \frac{\chi_{j}(m) \omega_{j}(m)}{m^{s}}, \quad j=1, \ldots, r,
$$

and denote by $P_{\underline{L}}$ the distribution of the $H^{r}(D)$-valued random element $\underline{L}(s, \omega, \underline{\chi})$, i.e.,

$$
P_{\underline{L}}(A)=m_{H}^{r}\left\{\omega \in \Omega^{r}: \underline{L}(s, \omega, \underline{\chi}) \in A\right\}, \quad A \in \mathcal{B}\left(H^{r}(D)\right) .
$$

Theorem 4.2. Suppose that $\left(\gamma_{1}, \ldots, \gamma_{r}\right) \in U_{r}$. Then $P_{T}$ converges weakly to $P_{\underline{L}}$ as $T \rightarrow \infty$. 
Pr o of. On a certain probability space with measure $\mu$, define a random variable $\xi_{T}$ and assume that $\xi_{T}$ is uniformly distributed on $\left[T_{0}, T\right]$. On the above probability space, define the $H^{r}(D)$-valued random element

$$
\underline{X}_{T, n}=\underline{X}_{T, n}(s)=L_{n}\left(s+\underline{\mathrm{i}} \underline{\left.\left(\xi_{T}\right), \underline{\chi}\right),}\right.
$$

and denote by $\underline{\hat{X}}_{n}=\underline{\hat{X}}_{n}(s)$ the $H^{r}(D)$-valued random element with distribution $V_{n}$, where $V_{n}$ is the limit measure in Lemma 2.2. Then Lemma 2.2 implies the relation

$$
\underline{X}_{T, n} \underset{T \rightarrow \infty}{\stackrel{\mathcal{D}}{\longrightarrow}} \underline{\hat{X}}_{n} .
$$

By Lemma 2.3, the family $\left\{V_{n}: n \in \mathbb{N}\right\}$ is tight, therefore, in view of the Prokhorov theorem 3: Theorem 6.1], it is relatively compact. Thus, there exists a subsequence $\left\{V_{n_{k}}\right\} \subset\left\{V_{n}\right\}$ weakly convergent to a certain probability measure $P$ on $\left(H^{r}(D), \mathcal{B}\left(H^{r}(D)\right)\right)$ as $k \rightarrow \infty$. This is equivalent to the relation

$$
\underline{\hat{X}}_{n_{k}} \underset{k \rightarrow \infty}{\stackrel{\mathcal{D}}{\longrightarrow}} P .
$$

Define one more $H^{r}(D)$-valued random element

$$
\underline{X}_{T}=\underline{X}_{T}(s)=\underline{L}\left(s+\underline{\mathrm{i}}\left(\xi_{T}\right), \underline{\chi}\right) .
$$

Then, using Lemma 3.2 , we find that, for every $\varepsilon>0$,

$$
\begin{aligned}
& \lim _{n \rightarrow \infty} \limsup _{T \rightarrow \infty} \mu\left\{\underline{\rho}\left(\underline{X}_{T}, \underline{X}_{T, n}\right) \geq \varepsilon\right\} \\
& \quad \leq \lim _{n \rightarrow \infty} \limsup _{T \rightarrow \infty} \frac{1}{\left(T-T_{0}\right) \varepsilon} \int_{T_{0}}^{T} \underline{\rho}\left(\underline{L}(s+\underline{\mathrm{i}} \underline{\gamma}(\tau), \underline{\chi}), \underline{L}_{n}(s+\underline{\mathrm{i}} \underline{\gamma}(\tau), \underline{\chi})\right) \mathrm{d} \tau=0 .
\end{aligned}
$$

This, 4.1, 4.2 and Proposition 4.1 show that

$$
\underline{X}_{T} \underset{T \rightarrow \infty}{\stackrel{\mathcal{D}}{\longrightarrow}} P
$$

i.e., $P_{T}$ converges weakly to $P$ as $T \rightarrow \infty$.

It remains to prove that $P=P_{L}$. The relation 4.3 shows that the measure $P$ is independent of the choice of the sequence $\left\{V_{n_{k}}\right\}$. Hence, we have that

$$
\underline{\hat{X}}_{n} \underset{n \rightarrow \infty}{\stackrel{\mathcal{D}}{\longrightarrow}} P
$$

or $V_{n}$ converges weakly to $P$ as $n \rightarrow \infty$. In [6], a discrete limit theorem for Dirichlet $L$-functions was discussed, and it was obtained that the limit measure $P$ of $V_{n}$, as $n \rightarrow \infty$, is $P_{\underline{L}}$. This remark and 4.3 complete the proof of the theorem.

Theorem 4.2 implies a limit theorem for the compositions $F(\underline{L}(s, \underline{\chi}))$.

TheOREM 4.3. Suppose that $\left(\gamma_{1}, \ldots, \gamma_{r}\right) \in U_{r}$, and $F: H^{r}(D) \rightarrow H(D)$ is a continuous operator. Then

$$
P_{T, F}(A) \stackrel{\text { def }}{=} \frac{1}{T} \text { meas }\{\tau \in[0, T]: F(\underline{L}(s+\underline{\mathrm{i}} \underline{\gamma}(\tau), \underline{\chi})) \in A\} \quad A \in \mathcal{B}(H(D)),
$$

converges weakly to $P_{\underline{L}} F^{-1}$ as $T \rightarrow \infty$.

Pro of. The theorem follows from Theorem 4.2, continuity of $F$ and 3 : Theorem 5.1]. 


\section{JOINT APPROXIMATION BY DIRICHLET L-FUNCTIONS}

\section{Support}

For proving of universality, we need the explicit form of the support of the measure $P_{\underline{L}}$. Since the space $H^{r}(D)$ is separable, the support $S_{P_{L}}$ of $P_{\underline{L}}$ is a minimal closed set of $H^{r}(D)$ such that $P_{\underline{L}}\left(S_{P_{\underline{L}}}\right)=1$. The set $S_{P_{\underline{L}}}$ consists of all $\underline{g} \in H^{r}(D)$ such that, for every open neighbourhood $\underline{G}$ of $\underline{g}$, the inequality $P_{\underline{L}}(\underline{G})>0$ is satisfied.

We recall that

$$
S=\{g \in H(D): g(s) \neq 0 \text { or } g(s) \equiv 0\} .
$$

Proposition 5.1. The support of $P_{\underline{L}}$ is the set $S^{r}$.

Proof. Let, for $\omega \in \Omega$,

$$
L(s, \omega, \chi)=\sum_{m=1}^{\infty} \frac{\chi(m) \omega(m)}{m^{s}}=\prod_{p}\left(1-\frac{\chi(p) \omega(p)}{p^{s}}\right)^{-1},
$$

and $P_{L}$ be the distribution of the $H(D)$-valued random element $L(s, \omega, \chi)$. Then it is well known, see, for example, 1], 11], that the support of $P_{L}$ is the set $S$. We will apply this remark for the support of $P_{\underline{L}}$.

Since the space $H^{r}(D)$ is separable, it is known that 3

$$
\mathcal{B}\left(H^{r}(D)\right)=\underbrace{\mathcal{B}(H(D)) \times \cdots \times \mathcal{B}(H(D))}_{r} .
$$

Therefore, it suffices to consider the measure $P_{\underline{L}}$ on the sets $A \in H^{r}(D)$ of the form

$$
A=A_{1} \times \cdots \times A_{r}, \quad A_{1}, \ldots, A_{r} \in H(D) .
$$

The Haar measure $m_{H}^{r}$ is the product of the Haar measures $m_{H, j}$ on $\left(\Omega_{j}, \mathcal{B}\left(\Omega_{j}\right)\right), j=1, \ldots, r$. Therefore,

$$
\begin{aligned}
P_{\underline{L}}(A) & =m_{H}^{r}\left\{\omega \in \Omega^{r}: \underline{L}(s, \omega, \underline{\chi}) \in A\right\} \\
& =\prod_{j=1}^{r} m_{H, j}\left\{\omega_{j} \in \Omega_{j}: L\left(s, \omega_{j}, \chi_{j}\right) \in A_{j}\right\}=\prod_{j=1}^{r} P_{L_{j}}\left(A_{j}\right),
\end{aligned}
$$

where $P_{L_{j}}$ is the distribution of the random element $L\left(s, \omega_{j}, \chi_{j}\right)$. Since, for all $j=1, \ldots, r$, the support of $P_{L_{j}}$ is the set $S$, the minimality of the support proves the proposition.

Proposition 5.2. Let $F: H^{r}(D) \rightarrow H(D)$ be a continuous operator such that, for every open set $G \subset H(D)$, the set $\left(F^{-1} G\right) \cap S^{r}$ is non-empty. Then the support of the measure $P_{\underline{L}} F^{-1}$ is the whole of $H(D)$.

Pr o of. Let $g \in H(D)$ be an arbitrary element, and $G$ its any open neighbourhood. Then the set $F^{-1} G$ is open as well, and contains an element of the set $S^{r}$. Thus, in view of Proposition 5.1. $F^{-1} G$ is an open neighbourhood of an element of the support of the measure $P_{\underline{L}}$. Hence,

$$
P_{\underline{L}} F^{-1}(G)=P_{\underline{L}}\left(F^{-1} G\right)>0 .
$$

Since $g$ and $G$ are arbitrary, this proves the proposition. 


\section{ANTANAS LAURINČIKAS — DARIUS ŠIAUČIŪNAS}

\section{Proof of universality}

We recall the Mergelyan theorem on the approximation of analytic functions by polynomials [13. Let $K \subset \mathbb{C}$ be a compact set with connected complements, and $f(s)$ be a continuous function on $K$ and analytic in the interior of $K$. Then, for every $\varepsilon>0$, there exists a polynomial $p(s)$ such that

$$
\sup _{s \in K}|f(s)-p(s)|<\varepsilon .
$$

Pro of of Theorem 1.3. By the Mergelyan theorem, there exist polynomials $p_{j}(s)$ such that

$$
\sup _{1 \leq j \leq r} \sup _{s \in K_{j}}\left|f_{j}(s)-\mathrm{e}^{p_{j}(s)}\right|<\frac{\varepsilon}{2} .
$$

Define the set

$$
G_{\varepsilon}^{r}=\left\{\left(g_{1}, \ldots, g_{r}\right) \in H^{r}(D): \sup _{1 \leq j \leq r} \sup _{s \in K_{j}}\left|g_{j}(s)-\mathrm{e}^{p_{j}(s)}\right|<\frac{\varepsilon}{2}\right\} .
$$

Then $G_{\varepsilon}^{r}$, in view of Proposition 5.1 , is an open neighbourhood of the element $\left(\mathrm{e}^{p_{1}(s)}, \ldots, \mathrm{e}^{p_{r}(s)}\right)$ of the support of the measure $P_{\underline{L}}$. Therefore

$$
P_{\underline{L}}\left(G_{\varepsilon}^{r}\right)>0 .
$$

Thus, Theorem 4.2 and the equivalent of weak convergence of probability measures in terms of open sets (3: Theorem 2.1]) show that

$$
\liminf _{T \rightarrow \infty} P_{T}\left(G_{\varepsilon}^{r}\right)>0
$$

This, 6.1 and the definitions of $P_{T}$ and $G_{\varepsilon}^{r}$ prove the first part of the theorem.

To prove the second part of the theorem, define one more set

$$
\hat{G}_{\varepsilon}^{r}=\left\{\left(g_{1}, \ldots, g_{r}\right) \in H^{r}(D): \sup _{1 \leq j \leq r} \sup _{s \in K_{j}}\left|g_{j}(s)-f_{j}(s)\right|<\varepsilon\right\} .
$$

Then the boundary $\partial \hat{G}_{\varepsilon}^{r}$ of $\hat{G}_{\varepsilon}^{r}$ lies in the set

$$
\left\{\left(g_{1}, \ldots, g_{r}\right) \in H^{r}(D): \sup _{1 \leq j \leq r} \sup _{s \in K_{j}}\left|g_{j}(s)-f_{j}(s)\right|=\varepsilon\right\},
$$

therefore, $\partial \hat{G}_{\varepsilon_{1}}^{r} \cap \partial \hat{G}_{\varepsilon_{2}}^{r}=\emptyset$ for different positive $\varepsilon_{1}$ and $\varepsilon_{2}$. From this remark, it follows that the set $\hat{G}_{\varepsilon}^{r}$ is a continuity set of the measure $P_{\underline{L}}$ for all but at most countably many $\varepsilon>0$. Thus, Theorem 4.2 and the equivalent of weak convergence of probability measures in terms of continuity sets ([3: Theorem 2.1$]$ ) imply the equality

$$
\lim _{T \rightarrow \infty} P_{T}\left(\hat{G}_{\varepsilon}^{r}\right)=P_{\underline{L}}\left(\hat{G}_{\varepsilon}^{r}\right)
$$

for all but at most countably many $\varepsilon>0$. In view of 6.1 , $G_{\varepsilon}^{r} \subset \hat{G}_{\varepsilon}^{r}$. Therefore, $P_{\underline{L}}\left(\hat{G}_{\varepsilon}^{r}\right)>0$ by 6.2. This, 6.3 and the definitions of $P_{T}$ and $\hat{G}_{\varepsilon}^{r}$ prove the second part of the theorem.

Proof of Theorem 1.4 By the Mergelyan theorem, there exists a polynomial $p(s)$ such that

$$
\sup _{s \in K}|f(s)-p(s)|<\frac{\varepsilon}{2} \text {. }
$$

Define the set

$$
G_{\varepsilon}=\left\{g \in H(D): \sup _{s \in K}|g(s)-p(s)|<\frac{\varepsilon}{2}\right\}
$$




\section{JOINT APPROXIMATION BY DIRICHLET L-FUNCTIONS}

Then, by Proposition 5.2, $G_{\varepsilon}$ is an open neighbourhood of the element $p(s)$ of the support of the measure $P_{\underline{L}} F^{-1}$. Therefore,

$$
P_{\underline{L}} F^{-1}\left(G_{\varepsilon}\right)>0 \text {. }
$$

From this, Theorem 4.3 and the equivalent of weak convergence of probability measures in terms of open sets, we obtain that

$$
\liminf _{T \rightarrow \infty} P_{T, F}\left(G_{\varepsilon}\right) \geq P_{\underline{L}} F^{-1}\left(G_{\varepsilon}\right)>0,
$$

and the definitions of $P_{T, F}$ and $G_{\varepsilon}$, and 6.4 prove the first part of the theorem.

Define one more set

$$
\hat{G}_{\varepsilon}=\left\{g \in H(D): \sup _{s \in K}|g(s)-f(s)|<\varepsilon\right\} .
$$

Then we have that this set is a continuity set of the measure $P_{L} F^{-1}$ for all but at most countably many $\varepsilon>0$. Therefore, Theorem 4.3 and the equivalent of weak convergence of probability measures in terms of continuity sets show that

$$
\lim _{T \rightarrow \infty} P_{T, F}\left(\hat{G}_{\varepsilon}\right)=P_{\underline{L}} F^{-1}\left(\hat{G}_{\varepsilon}\right)
$$

for all but at most countably many $\varepsilon>0$. Moreover, in view of 6.4 , we have that $G_{\varepsilon} \subset \hat{G}_{\varepsilon}$. Therefore, by 6.5), the inequality $P_{\underline{L}, F^{-1}}\left(\hat{G}_{\varepsilon}\right)>0$ holds. This together with 6.6 proves the second part of the theorem.

\section{REFERENCES}

[1] BAGCHI, B.: The Statistical Behaviour and Universality Properties of the Riemann Zeta-Function and other Allied Dirichlet Series, Ph. D. Thesis, Indian Stat. Institute, Calcutta, 1981.

[2] BAGCHI, B.: A joint universality theorem for Dirichlet L-functions, Math. Z. 181 (1982), 319-334.

[3] BILlingSLEY, P.:. Convergence of Probability Measures, Wiley, New York, 1968.

[4] BOHR, H.: Über das Verhalten von $\zeta(s)$ in der Halbebene $\sigma>1$, Nachr. Akad. Wiss. Göttingen II Math. Phys. Kl. (1911), 409-428.

[5] BOHR, H.-COURANT, R.: Neue Anwendungen der Theorie der Diophantischen Approximationen auf die Riemannsche Zetafunktion, J. Reine Angew. Math. 144 (1914), 249-274.

[6] DUBICKAS, A.-LAURINČIKAS, A.: Joint discrete universality of Dirichlet L-functions, Arch. Math. 104 (2015), 25-35.

[7] GONEK, S.M.: Analytic Properties of Zeta and L-functions, Ph. D. Thesis, University of Michigan, 1979.

[8] KACZOROWSKI, J.-LAURINČIKAS, A.-STEUDING, J.: On the value distribution of shifts of universal Dirichlet series, Monatsh. Math. 147(4) (2006), 309-317.

[9] KARATSUBA, A. A.-VOROnIN, S. M.: The Riemann Zeta-Function, Walter de Gruyter, Berlin, New York, 1992.

[10] LAURINČIKAS, A.: Limit Theorems for the Riemann Zeta-Function, Kluwer Academic Publishers, Dordrecht, 1996.

[11] LAURINČIKAS, A.: On joint universality of Dirichlet L-functions, Chebyshevskii Sb. 12(1) (2011), 124-139.

[12] LAURINČIKAS, A.-GARUNKŠTIS, R.: The Lerch Zeta-Function, Kluwer Academic Publishers, Dordrecht, 2002.

[13] MERGELYAN, S.N: Uniform approximation to functions of a complex variable, Usp. Matem. Nauk 7(2) (1952), 31-122 (in Russian); [Amer. Math. Soc. Translation vol. 101, 1954, p. 99].

[14] NAKAMURA, T.: The joint universality and the generalized strong recurrence for Dirichlet L-functions, Acta Arith. 138 (2009), 357-362.

[15] NAKAMURA, T.-PAŃKOWSKI, Ł.: Erratum to: The generalized strong recurrence for non-zero rational parameters, Archivum Math. 99 (2012), 43-47.

[16] PAŃKOWSKI, Ł.: Some remarks on the generalized strong recurrence for L-functions. In: New Directions in Value Distribution Theory of Zeta and L-Functions, Shaker Verlag, Aachen, 2009, pp. 307-315. 
[17] PAŃKOWSKI, Ł.: Joint universality and generalized strong recurrence with rational parameter, J. Number Theory 163 (2016), 61-74.

[18] PAŃKOWSKI, Ł.: Joint universality for dependent L-functions, Ramanujan J. 45 (2018), 181-195.

[19] STEUDING, J.: Value-Distribution of L-Functions. Lecture Notes in Math. 1877, Springer, Berlin, 2007.

[20] VORONIN, S. M.: The distribution of the non-zero values of the Riemann zeta-function, Trudy Matem. Inst. Steklov 128 (1972), 131-150 (in Russian).

[21] VORONIN, S. M.: Theorem on the "universality" of the Riemann zeta-function, Izv. Akad. Nauk SSSR, Ser. Mat. 39 (1975), 475-486 (in Russian); [Math. USSR Izv. 9 (1975), 443-453].

[22] VORONIN, S. M.: On the functional independence of Dirichlet L-functions, Acta Arith. 27 (1975), 493-503 (in Russian).

Received 14. 11. 2020

Accepted 3. 2. 2021

\author{
* Institute of Mathematics \\ Faculty of Mathematics and Informatics \\ Vilnius University \\ Naugarduko str. 24 \\ LT-03225 Vilnius \\ LITHUANIA \\ E-mail: antanas.laurincikas@mif.vu.lt \\ ** Institute of Regional Development \\ Siauliai Academy \\ Vilnius University \\ P. Višinskio str. 25 \\ LT-76351 Šiauliai \\ LITHUANIA \\ E-mail: darius.siauciunas@sa.vu.lt
}

